\title{
Multilinguales
}

Multilinguales

$14 \mid 2020$

Enseignement universitaire et professionnalisation : enjeux, questionnements et défis socio-économiques

\section{Traduire le couple texte-image du point de vue de la paratraduction : cas de la bande dessinée}

Translating the text-image couple from th perspective of paratranslation: case of comic books

\section{Badra Ramdani}

\section{(2) OpenEdition}

Journals

Édition électronique

URL : https://journals.openedition.org/multilinguales/5835

DOI : $10.4000 /$ multilinguales.5835

ISSN : 2335-1853

Éditeur

Université Abderrahmane Mira - Bejaia

\section{Référence électronique}

Badra Ramdani,

"Traduire le couple texte-image du point de vue de la paratraduction : cas de la bande dessinée », Multilinguales [En ligne], 14 | 2020, mis en ligne le 10 décembre 2020, consulté le 02 juillet 2021. URL: http://journals.openedition.org/multilinguales/5835; DOI : https://doi.org/10.4000/multilinguales. 5835

Ce document a été généré automatiquement le 2 juillet 2021.

\section{(i) $\$$

Multilinguales est mise à disposition selon les termes de la Licence Creative Commons Attribution Pas d'Utilisation Commerciale - Pas de Modification 4.0 International 


\title{
Traduire le couple texte-image du point de vue de la paratraduction : cas de la bande dessinée
}

Translating the text-image couple from th perspective of paratranslation: case of comic books

\author{
Badra Ramdani
}

1 La bande dessinée ou la littérature graphique (Thierry Groensteen, 2005), se propose comme un genre de narration à forte présence du visuel, misant sur le verbal et le non verbal. Sur la langue de ses personnages braves, surnaturels, drôles ou comiques, la BD régale ses lecteurs de tout âge, de ses récits relevant du fictif comme de l'effectif. Dessinateurs et auteurs collaborent, chacun en ce qui le concerne, à la création de ce neuvième art, qui pour assurer une bonne compréhension et consommation du produit final, exige du lectorat certaines compétences notamment celles relevant de la manière de le lire. Visible et lisible se rangeant dans un espace le plus économiquement possible, tissent entre eux une relation intersémiotique, appelée "couple texteimage ».

Cette entité iconotextuelle habitant les vignettes tout au long de la narration, constitue l'essence même du sens à comprendre, et par conséquent, nécessite un certain mode de " voir », d'interpréter et de traduire. La bande dessinée, vu ses particularités, engage le traducteur au-delà du texte qu'il a l'habitude de traduire. Or, la traductologie, cette science s'ouvrant sur une multiplicité de pistes de recherches appelle le traducteur à aiguiser ses compétences à chaque fois besoin est et met à sa disposition et de façon systématique des outils d'exploration lui permettant une meilleure traduction.

2 La paratraduction en l'occurrence, issue du croisement de la notion de paratexte du propre Genette et la sémiologie de l'image, offre au traducteur un moyen de dépistage de tout champ combinant textes à images. La traductologie la considère comme un « concept clé pour traduire le couple texte-image » (Frias, 2010 : p. 295). Le traducteur de la bédé qui est censé traduire et le texte et l'image de chaque vignette, doit tout d'abord comprendre le sens que recèle cette combinaison iconotextuelle comme étant 
un tout complet. De ce fait comment la notion de pararaduction pourrait-elle aider le traducteur à déceler ce sens en premier lieu et le traduire le plus correctement possible en deuxième lieu ? Traduirait-il le texte et l'image du même couple l'un séparément de l'autre ou devrait-il prendre en considération le type de relation qu'ils entretiennent pour former ce couple? Nous tenterons de répondre à ces questions à travers notre article que nous structurons comme suit: notre premier axe porte sur la bande dessinée, objet de notre étude, puis nous nous étalons sur la notion de paratraduction telle qu'elle est conçue par son créateur José Yusté Fias, en deuxième axes, nous concluons enfin par examiner l'importance de la paratraduction dans la lecture et la traduction du couple texte-image, en l'appliquant sur un numéro de la bande dessinée «les schtroumpfs noirs» en français et sa traduction en anglais, en guise d'étude constative.

\section{La bande dessinée}

3 La bande dessinée est pour Yve Frémion (1990) une narration figurative (ou le récit en images), elle se définit aussi comme une suite d'images organisées pour raconter une histoire comique ou réaliste (Alain. I, 2017), ou encore elle est «cet art hybride, résultant, en effet, du croisement du texte et de l'image sur le même support imprimé " (Caraballo, 2016: p. 46). Chacune de ces définitions souligne l'un des paramètres régissant la bande dessinée, à savoir : la technique de conjugaison de textes aux images, le support matériel sur lequel est imprimée cette narration textuelle et visuelle et aussi les thèmes de narration qui décident de son genre. (Ibid, p. 48). Somme toute, la bande dessinée se profile comme un art de narration en vignettes, à l'intérieur desquelles, texte et images construisent ensemble le sens du message à transmettre. La lecture séquentielle qu'exige la bande dessinée demande du visiolecteur ${ }^{1}$ la connaissance de ses codes et caractéristiques pour garantir une meilleure réception.

\section{Déchiffrer la bande dessinée}

4 La bande dessinée possède des particularités en la forme et sur le fond, il nous paraît alors judicieux de les survoler dans le but de non seulement s'y rendre compte mais aussi se préparer pour une lecture correcte et une compréhension optimale.

\subsection{Morphologie de la BD}

5 La bande dessinée tout comme le livre, est lue de gauche à droite et de haut en bas, planche par planche, dans la mesure où planche est équivalent de page. Une planche se compose de plusieurs bandes qui se superposent en largeur. Une bande est une suite de vignettes ou de « cases » qui se rangent horizontalement. La vignette quant à elle, se meuble de dessins, de bulles et de cartouches. Les dessins figurent des personnages dans leurs scènes, des petits dessins qu'on appelle "idéogrammes ou signes ", qui mettent en relief l'expression des personnages, et aussi des lettres dessinées qu'on appelle " onomatopées ». Les bulles ou "phylactères » sont l'espace qui contient les propos des personnages, généralement des dialogues, et enfin des cartouches qui avec leurs aspects tantôt narratifs, tantôt descriptifs, marquent brièvement le changement de lieu ou de temps². 


\subsection{Sur le fond}

La bande dessinée nonobstant son aspect "gigogne " laisse discerner ses trois particularités de fond : visualité, scripturalité, et oralité.

\subsubsection{Visualité}

7 Comme son nom d'indique, le dessin prime dans la BD, sa visualité ne peut donc être passée inaperçue, Will Eisner par exemple le souligne dans sa définition de la BD dans ces propos : «média visuel composé de séquences d'images » (1998: p. 4). L'image toute éloquente, vu qu' e elle est légitimement considérée comme un texte au sens fort du terme » (Frias, 2017), raconte des histoires qui s'offrent plutôt à la vue qu'à l'esprit, en mobilisant toutes ses valeurs graphiques iconiques et plastiques. Or, « une image seule n'est pas une bande dessinée [...] ce qui compte, c'est que plusieurs images soient réunies dans une même séquence signifiante » (Pascal Krajewsky, 2016), ce qui traduit l'appellation «bande », d'où sa définition en tant qu'art séquentiel.

Dans la bande dessinée, les images sont vues non seulement dans l'espace qui leur est imparti, soit les vignettes, mais aussi dans leur montage sur toute la bande qui les agence, et encore dans tout l'espace de la planche qui accueille ces bandes. (Ibid). Les images sont donc perçues et lues dans la globalité de leur rangement sur la surface de l'album et aussi dans leur agencement avec le linguistique qui les accompagne.

\subsubsection{Scripturalité}

8 C'est l'aspect textuel de la bande dessinée. Très réduit « en quantité » et non pas en qualité, dans l'intention de respecter la condition sine qua non de l'économie dans la bande dessinée. "Le texte est cet élément matériel qui fait partie de l'esthétique générale du strip » (Harry Morgan, 2015) mais aussi de la trame narrative. L'esthétique du texte sous-entend la beauté du discours dans son contenu (le beau langage), comme elle sous-entend la beauté dans la forme (le style de la police qui n'est aucunement anodin). Le style de la police rime harmonieusement avec le type du discours, et dont les connotations transparaissent sur son aspect visuel, à l'instar de l'exemple cidessous :

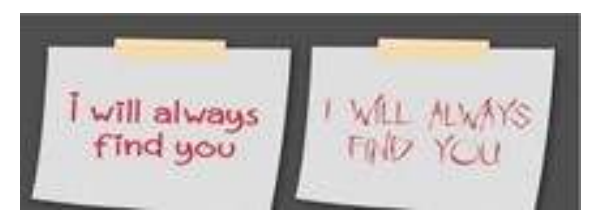

9 Le message «I will always find you » connote dans la première étiquette, un message affectueux qu'aurait laissé un amoureux à sa partenaire ou le contraire. Le même message dans la deuxième étiquette donne froid dans le dos, et laisse comprendre qu'on est sujet de menace ou de péril imminent. La scripturalité jalonne la trame narrative. Elle trace tantôt les dialogues qu'échangent les personnages dans la bédé, tantôt leurs pensées, comme elle peut transcrire des cartouches ${ }^{4}$ ou des récitatifs ${ }^{5}$ sans toutefois oublier les titres. Le texte sert à exprimer et représenter certaines actions pratiquement impossibles à rendre par l'image. Le texte est donc le parler de la bande dessinée et sa « voix » (Pascal Krajewsky, 2016). 


\subsubsection{Oralité}

10 Comme la bande dessinée nous "présente un récit presque entièrement raconté sous forme de dialogues, si l'on excepte les quelques récitatifs » elle s'avère " extrêmement sonore " (Catherine Delesse, 2001: p. 321). Cette oralité est l'effet que produisent de nombreux procédés graphiques qui entourent les personnages et incarnent toute « la didascalie » de la bande dessinée. Dans la bande dessinée tout parle, la taille de la police $\mathrm{du}$ texte joue le rôle d'indicateur du volume de la voix des personnages, écrire les paroles en grande ou en petite taille indique si l'on crie ou l'on chuchote, les attributs de caractères (gras, italique, souligné) ajoutent du ton aux paroles des personnages, une conversation en gras atteste de la colère de ses interlocuteurs. Les signes de ponctuation eux aussi prennent du sens dans la BD, une suite de points d'interrogation et d'exclamation exprime le sentiment de colère, d'étonnement ou de perplexité qu'aurait envahi le personnage qui parle. (Ibid, p. 322)

11 Il existe encore un élément fréquemment employé dans la bande dessinée pour ajouter de l'accent, selon le contexte, aux paroles des personnages, il s'agit des signes ou des idéogrammes. Une bulle saupoudrée de notes de musique fait comprendre que le personnage fredonne ou donne du rythme à ses paroles, les cœurs et des fleurs quant à eux, donnent un timbre mielleux à la voix qui parle.

Les bulles encadrant les paroles des personnages, contribuent elles aussi à oraliser la bande dessinée. Les différentes formes que prennent les bulles parlent. Un ballon nuage indique que l'on songe, celui en forme d'éclair indique qu'on crie, et celui en forme de fumée ou bulle fantôme atteste de la peur'. L'onomatopée est "Un autre élément sonore » qui caractérise la bande dessinée. Ces codes monosyllabiques «traduisent les différents bruits, chocs, coups, chutes, portes qui grincent, sonneries de téléphones, etc. » (Catherine Delesse, $2001:$ p. 326). Variées d'un pays à un autre, les onomatopées sont considérées comme un texte dessiné parce qu'elles obéissent aux techniques de déformation et de coloration selon le sens. «Bleuuurp " par exemple est l'onomatopée qui imite le son de vomissement, la couleur verte renforce le sens du son.

Il est à noter qu'au demeurant, tout se joue autour du graphisme, écrit et/ou dessiné. Les deux modes d'expression sont à la fois vus et lus. Or, leur mode de lecture dans leur ensemble est tout particulier, car texte et image s'épousent pour devenir un « textimage $»^{7}$, un couple qui tisse différents liens ou rapports qu'il faut analyser.

\section{Analyser la bande dessinée}

13 La particularité iconotextuelle de la bande dessinée a intéressé beaucoup de chercheurs et théoriciens qui chacun selon son point de vue, ont contribué à mieux maîtriser sa lecture et sa compréhension, et ce par la proposition de grilles d'analyse que nous citons par ordre chronologique.

Pierre Fresnault-Duruelle, en tant que sémiologue, propose au courant des années 70s une manière d'analyse de la $\mathrm{BD}$, il s'agit d'étudier les signifiants selon une sémiologie de cases, de bulles, et de relations texte-image, avec une " élaboration d'une symétrie en accord avec le signifié globale de la narration » (Fresnault-Dureuelle, 1976: p. 17. Cité par Saâd-Eddine Fatmi, 2011: p.92). De son côté, Pierre Masson (1985), base sa contribution sur la linguistique, et opte pour une lecture de la BD dans deux sens complémentaires: vertical "qui rattache chaque élément de l'image, considéré 
isolément, à un réservoir de signification constitué aussi bien par la tradition picturale que par les conventions morales et sociales de l'époque présente » (Masson, $1985:$ p. 13. Cité par Ibid), et horizontal qui prend la vignette ou la planche « comme un puzzle dont les pièces tirent leur signification de la manière selon laquelle elles sont juxtaposées ". (Ibid). Les années 90s sont marquées par les travaux de Tilleul/Vanbraband/Marlet inspirés de la sémiotique narrative de Greimas. Ils proposent deux niveaux de lecture de la $\mathrm{BD}$, « la première est élémentaire, plate et dénotée, aboutissant à un résumé, une formalisation du récit» (Tilleul/Vanbraband/Marlet, 1991 : p. 46. Cité par Ibid). C'est la grammaire du récit. La deuxième lecture porte sur la grammaire du discours en impliquant l'étude des aspects graphiques de la BD et l'analyse des réseaux de sens supplémentaires qu'ils créent (Ibid).

Benoît Peeters (1991) axe son étude sur la « case, le strip, la planche, les relations entre le texte et le dessin, entre le scénario et sa mise en images " (Peeters, 1984: p. 7. Cité par Ibid, p. 93). La méthode de Thierry Groensteen (2000) analyse la BD en tant qu'un ensemble original « de mécanisme producteurs de sens » (Groensteen, 1999: p. 2), chez lui l'image prime et l'unité d'analyse est la case ou la vignette qui conjugue les aspects visuels et discursifs. Enfin Harry Morgan, propose une approche stripologique qui selon lui «porte sur l'étude des littératures dessinées » (Morgan, 2003 : p. 375), la notion de littérature dessinée constitue "l'interaction entre les contraintes physiques du médium et le contenu » (Ibid), ce qui sous-entend l'étude de la relation qu'entretient la forme de la bédé dans son dispositif physique et son contenu narratif.

Toutes les grilles d'analyse, nonobstant les différents points de départ, tiennent compte du caractère binaire de la $\mathrm{BD}$, d'où les dichotomies signifiant-signifié, verticalhorizontal, littérature-dessinée et couple ou relation texte-image. Somme toute, la BD est lue dans sa globalité et avec prise en considération de toutes ses caractéristiques.

\section{Traduire la bande dessinée}

Le traducteur de la BD sait bien qu'il est devant une tâche aussi passionnante que contraignante. Pour assouvir sa passion et gérer sa contrainte à la fois, il doit puiser de "son système sous-jacent de connaissances déclaratives et essentiellement opérationnelles nécessaires pour traduire» (Hurtado Albir, 2008: p.27. Cité par Comitre Narvaez, 2015: 132) qui constitue ses compétences traductionnelles. Ces compétences qui se décomposent en plusieurs sous-compétences: «bilingue, extralinguistique, traductionnelle (connaissances en traduction), instrumentale, stratégique et psychophysiologique» (Ibid: 133), viennent en aide au traducteur à chaque besoin est et tout au long du processus de traduction.

Si le sens est l'essence de toute l'opération de traduction, le traducteur, et à l'effet de mieux le comprendre et mieux le traduire, doit de prime abord déterminer le cadre théorique dans lequel s'inscrivent ses démarches traductives. S'agissant en l'occurrence, d'un texte accompagné de tout un appareil paratextuel, la notion de paratraduction s'impose.

\subsection{Paratraduire la bande dessinée}

17 La paratraduction est récemment créée en traductologie à l'initiative de José Yuste Frias $^{8}$, qui en 2004 s'est rendu compte de l'importance des éléments paratextuels qui 
accompagnent le texte proprement dit, l'entourent, le présentent et l'introduisent. Selon lui la paratraduction est « l'analyse des productions verbales, iconiques ou verboiconiques ou simplement des productions matérielles des paratextes à paratraduire ou paratraduits » (Frias, 2010 : p. 290). L'objet de la paratraduction est le paratexte qui est pour Gérard Genette cet ensemble d'éléments hétérogènes contournant le texte, et porteurs de sens (1987), et qui fait objet de traduction. Tout comme le texte qui fait objet d'une traduction, et qui passe de son état "original » à l'autre "traduit », de même, le paratexte qui accompagne ce texte, passe d'un état "original» à celui " paratraduit » dans ce sens, la paratraduction s'accomplit en deux étapes. En amont, c'est l'analyse du paratexte avant toute opération de traduction et en aval, c'est la transposition du paratexte de la langue de départ dans la langue d'arrivée, compte tenu " des pouvoirs et des enjeux esthétiques, politiques, idéologiques, culturels et sociaux véhiculés par les différentes productions paratextuelles » (José Frias, 2010 : p. 192).

La paratraduction ne se limite pas aux seuls éléments verbaux ou linguistiques du paratexte, elle se focalise aussi sur ceux non verbaux comme les illustrations (images) et leur accorde toute importance dans les recherches traductologiques. Etudier le linguistique dans sa relation avec l'iconique, constitue l'objectif le plus éminent de la paratraduction, c'est l'étude et l'analyse du couple texte-image.

\subsection{Traduire le couple texte-image dans la BD}

18 En vue de traduire les couples texte-image qui fourmillent dans la bande dessinée, il convient de prime abord de déterminer le type de relation qu'entretient le texte avec l'image du même couple. Sophie Van Der énumère trois types: "texte et image n'entrent en relation que de trois manières. Car le champ des possibilités est finalement rapidement circonscrit : texte et images peuvent-ils faire autre chose que se répéter, se compléter ou se contredire ?» (2006 : 120, Cité par Marc Parayre, 135).

Il est à noter que toutes les recherches qui se sont intéressées au couple (texte/image) de la BD, l'ont tout le temps écrit avec une barre entre le texte et l'image, ce qui traduit l'état de belligérance qu'existait entre ces deux entités. Certains chercheurs prévalent le texte au détriment de l'image, comme Flaubert qui explique que

la plus belle description littéraire est dévorée par le plus piètre dessin [...] Une femme dessinée ressemble à une femme, voilà tout. L'idée est dès lors fermée, complète, et toutes les phrases sont inutiles, tandis qu'une femme écrite fait rêver à mille femmes. Donc ceci étant une question d'esthétique, je refuse formellement toute espèce d'illustration. (Flaubert, 1980 : p. 122. Cité par Marc Parayre, p. 132),

d'autres prétendent que l'image prime le texte « un bon dessin vaut mieux qu'un long discours ». (Ibid).

La paratraduction commence alors par supplanter la barre et la remplacer par un trait d'union, en guise de refuser l'idée théorique que l'une des parties du couple prime l'autre, ou que l'une entrave l'autre.

José Yuste Frias affirme que «le verbal et le visuel entretiennent toujours des relations étroites voire métisses dont il faut savoir bien lire et interpréter l'interaction intersémiotique pour mieux la traduire» $(2010:$ p. 299). Le texte devient image et l'image devient texte dans une relation harmonieuse indissociablement et fructueusement créatrice de sens. Frias ajoute que

Dans toute traduction spécialisée de textes à images, le traducteur ne traduit jamais d'une langue à une autre, isolées de tout autre code sémiotique [...] le traducteur 
doit lire, interpréter et traduire non seulement tous les éléments textuels mais aussi tous les éléments paratextuels qui composent l'imaginaire présent dans chaque entité iconotextuelle formée par le couple texte-image (2011 : p 269).

Le traducteur doit être averti de la dimension culturelle et sociolinguistique que renferment ces couples texte-image et qui constitue dans la majorité des cas l'essence même du sens à traduire.

\section{Etude de cas}

21 Dans ce volet, nous proposons une étude contrastive sur notre corpus qui porte sur l'une des icônes culturelles de la bande dessinée " les Schtroumpfs ». Nous tenterons de paratraduire le couple texte-image dans ses deux versions, originale en Français «Les Schtroumpfs Noirs » et sa traduction en Anglais « The Purple Smurfs ». Notre étude se voulant qualitative, l'exemple que nous choisissons à soumettre à l'épreuve de la paratraduction est le couple texte-image qui se trouve sur la première de couverture de l'album des deux versions. La raison derrière notre choix est cette importance qu'occupe ce couple qui, non seulement résume tout l'épisode des schtroumpfs noirs dans les détails le plus nécessaires, mais aussi qui parle de tous les couples qui racontent l'histoire au sein des vignettes, dans leur aspect formel et visuel.

\subsection{Présentation du corpus}

Pierre Culliford connu sous son nom de plume Peyo est le créateur des Schtroumpfs, "un peuple imaginaire de petites créatures humanoïdes bleues », ils habitent des maisons en forme de champignons quelque part au milieu d'une forêt en Europe, exactement dans le pays dit «Schtroumpf » qui est pratiquement difficile à repérer. Outre leur caractère espiègle, les Schtroumpfs ont un langage bien particulier, ils parlent «Schtroumpf ». Le monde de fiction que leur a crée Peyo leur permet de vivre en toute paisibilité et joie sauf dérangés par leurs pires ennemies Gargamel et son chat Azraël. (Super prof).

«Les Schtroumpfs noirs » est la première histoire de la série «Les Schtroumpfs » elle est publiée pour la première fois dans le no 1107 du journal Spirou sous forme de minirécit, puis redessinée et publiée dans l'album du même nom en 1963.

\subsection{Traduire le couple texte-image dans la BD}

Le texte qui constitue la première partie du couple de la première de couverture est en l'occurrence le titre, la preuve en est que Gérard Genette le considère comme « un micro texte », et précise qu'il est « un texte à propos d'un texte » (1987).

Le titre « les schtroumpfs noirs » est écrit en noir, en grand et en gras, et s'étale sur le premier tiers de l'image qui l'accompagne et qui prend toute la surface de la première de couverture. L'illustration figure deux lutins, l'un noir aux yeux rouges surgissant des bois, ayant l'air d'être en colère et regarde méchamment un autre lutin bleu, une fleur rouge (un coquelicot) à la main, avec un air très paisible et semble être non averti du danger qui le guette. 


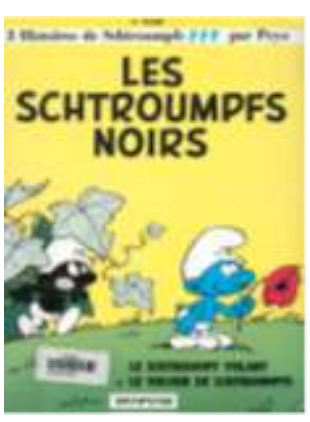

Le couple objet de la présente analyse ressemble à tous les autres couples texte-image des vignettes de la $\mathrm{BD}$. L'image contient le texte, ce dernier occupe un espace réduit par rapport à l'image comme c'est rituel en $\mathrm{BD}$, mais aucune bulle ne le renferme. Cela peut être expliqué par le fait que ce texte qui est à l'origine le titre de la BD, contient en lui tous les détails de l'histoire et parle « sur la langue » de toutes les bulles qui racontent l'intrigue dedans la couverture, il ne devrait pas dans ce sens être conditionnée dans une bulle et devrait rester en revanche dans l'espace libre. Selon l'approche titrologique, le titre "les schtroumpfs noirs» est thématique, du moment "qu'il emprunte de l'univers de l'histoire un élément le caractérisant» (Djaouida, 2011: p. 36), les personnages en l'occurrence « les schtroumpfs ». Ce nom masculin pluriel est qualifié d'un adjectif «noirs ", or, Peyo a habitué le lecteur franco-belge au fait que ses lutins sont d'origine de couleur bleue. Le titre vient renforcer sa littéralité 9 par son aspect chromatique de couleur noire, ce qui produit un effet très interpellant, que s'estil arrivé aux schtroumpfs bleus et pourquoi ils deviennent noirs?

L'image quant à elle, précise de prime abord les éléments spatio-temporels de l'histoire. Il s'agit de plein jour traduit par la couleur jaune de l'aplat de la première de couverture, et sur la terre du pays "Schtroumpfs", (la forêt) représentée par l'herbe verte et rase. Ensuite, et suivant le sens de lecture de l'image de gauche à droite, la narration figurative annonce directement l'évènement qui vient perturber la paisibilité du monde des schtroumpfs. Un schtroumpf noir surgit des ténèbres des bois épais, tout furieux, les sourcils froncés, les yeux étincellent de colère rouge, les dents grincent de rage, sur le bout d'attaquer sans pitié et au dépourvu un schtroumpf bleu, celui-ci se tient debout à droit en toute sérénité, visage aux traits attestant de la bonté et le sourire témoignant de la béatitude, ce qui symbolise la réalité de tous les schtroumpfs bleus qu'ont lus les lecteurs franco-belges.

Il est important de rappeler que la couleur noire en Occident, est associée au deuil, à la tristesse, au désespoir, à l'inconnu, à la peur et à la mort. (Série, 2016). Personne ne peut oublier le deuil qui a envahit l'Europe à cause de cette maladie dangereuse qui est devenue épidémique voire pandémique et qui a tué la quasi totalité de la population européenne «la peste », appelée aussi la peste noire. La piqure d'une puce infecté de la bactérie est à l'origine de la transmission horriblement rapide entre les personnes. Il semble que Peyo s'est servi de tous ces détails pour importuner ses lutins infortunés dans cette série.

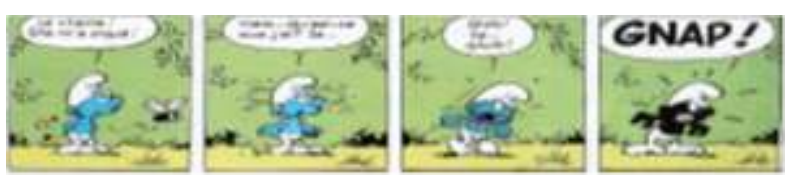

(Peyo, les schtroumpfs noirs, 1963 : p. 5) 


$$
\text { est }
$$
En médecine, cette fleur contient un alcaloïde particulier qui a des effets et des
propriétés sédatifs et servait à calmer la nervosité. (Langage fleur. Com). Le coquelicot Le coquelicot que tient le schtroumpf bleu dans sa main en guise de l'offrir à quelqu'un est très symbolique. Selon la mythologie grecque ${ }^{10}$, le coquelicot est une fleur qui fleur et plante médicinale est l'élément de dénouement de l'histoire. D'une part, son espèce fait référence à la plante thérapeutique qui a servi de trouver un remède aux schtroumpfs contaminés et arrêter la contagion. D'autre part, sa couleur rouge symbolise la guerre, la lutte mais aussi la joie, la santé et le triomphe (Canalblog, 2006) ${ }^{11}$. Toutes ces connotations sont dénotées par les évènements qui déclinent vers leur fin suivant le même ordre du symbolique du rouge, la « lutte » entre les deux clans, les schtroumpfs enragés «noirs» et les schtroumpfs sains «bleus», le triomphe des bleus par la découverte du traitement, leur rétablissement et la reprise de leur santé et enfin l'expression de leur joie. Aussi l'emplacement du coquelicot vers l'extrême droite de l'image connote la façon dont est achevée l'histoire. Le coquelicot calme la fureur des schtroumpfs noirs et les réconcilie avec les schtroumpfs bleus ou plus exactement avec leur origine avant la contamination, annonçant ainsi la fin par le retour au calme et à la paisibilité du début de l'histoire.

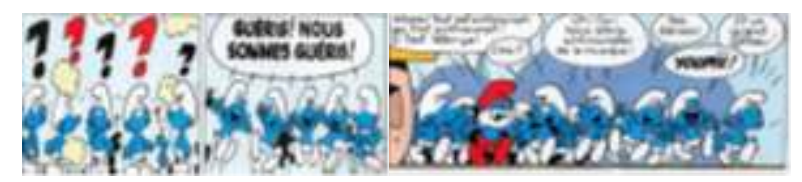

(Peyo, les schtroumpfs noirs, 1963 : pp. 21-22)

Texte et image du couple sur la première de couverture de la version originale entretiennent une relation de complémentarité et de circularité, le texte connote des questions, l'image les dénote, les deux résument l'histoire chacun dans son langage, et construisent ensemble le sens à comprendre.

\subsection{The Purple smurfs}

Le conte des schtroumpfs noirs a fait sa première parution aux Etat Unis à travers le huitième art, dans la série télévisée d'animation produite dans les années 1980 par Hanna-Barbera, où les schtroumpfs noirs sont devenus pourpres (violets). Ce n'est qu'en 2010 que la version américaine de l'album a finalement été publiée, sous le titre « The purple Smurfs ». 


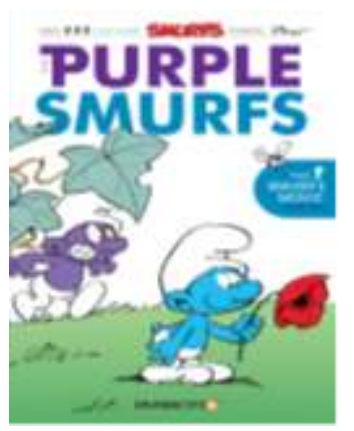

Dans le couple texte-image de la première de couverture de la version traduite, le titre est traduit en respectant la forme syntaxique du titre original, (un déterminent + un nom + un adjectif). Toutefois, l'adjectif qualifiant les «smurfs» devient pourpre dans ses aspects linguistique et chromatique, alors que le nom «smurfs » est toujours en bleu. Le tout sur un aplat blanc assorti. L'image, quant à elle, est reprise comme telle qu'elle est dans la version française, dans tous ses détails, sauf la couleur du schtroumpf noir qui devient pourpre. Un petit détail de valeur vient s'ajouter sur l'image, la mouche Bzz qui, ayant les même symptômes du schtroumpf pourpre, vient s'installer sur le panneau de publicité qui annonce la diffusion très prochaine de l'œuvre cinématographie les « Smurfs».

31 La première question qui se pose est celle relevant de la couleur, pourquoi le pourpre? $\mathrm{Au}$ fait, choisir changer la couleur des lutins franco-belges n'est aucunement anodin. Cette couleur pourpre est citée dans l'Antiquité comme provenant d'une substance animale (code couleur) d'un rouge violacé, ce qui explique l'origine de l'épidémie dans le déroulement des évènements et aussi sur la première de couverture "la mouche ». En outre, le pourpre symbolise le deuil en Amérique du Sud (Epson), tout comme est la couleur noire en Europe. En pathologie, il existe une maladie qui correspond au pourpre, c'est justement la maladie " purpura $~^{12}$, et exactement le purpura infectieux. Une maladie infectieuse qui infecte la peau et la rend de couleur pourpre. Tant de raisons motivent le choix du pourpre pour représenter cette épidémie due à la contamination par morsure, dans le contexte américain.

L'aspect chromatique bicolore du titre n'est pas à l'abri d'une intention bien déterminée. S'agissant d'une traduction, l'album s'adresse cette fois-ci aux lecteurs étrangers à la culture de l'original. Il est alors judicieux de leur préciser la couleur originale en misant sur l'aspect chromatique du mot " smurfs ", pour transmettre l'idée que les schtroumpfs sont originairement bleus, mais qui sont devenus "purple " à cause de l'épidémie, et ce selon le même jeu de couleur sur le mot pourpre. Le couple texte-image paratraduit dans l'anglais mise sur le code chromatique pour donner une couleur locale de la langue d'arrivée, les précisions ajoutées s'avèrent utiles pour assurer une bonne compréhension chez le lecteur américain. Texte et image entretiennent une relation explicative, le jeu de couleur est le même dans les deux entités du couple qui se sont entraidées pour bien rendre le sens de l'original dans la traduction.

Le traducteur de la BD est censé comprendre que la BD « est un moyen d'expression, un véhicule de la culture de masse et son idéologie mais aussi un art» (Baron-Carvais, 1985: p.122), et comme tel, il devrait se rendre compte de sa particularité iconotextuelle qui se traduit par la forte présence des couples texte-image depuis la première de couverture jusqu'à la quatrième de couverture de l'album. Le traducteur 
devrait alors actualiser et adapter ses compétences à l'effet de surmonter les contraintes qui y sont imposées. Paratraduire demande au traducteur une vigilance quant aux prouesses du texte et de l'image et la relation qu'ils tissent entre eux.

\section{BIBLIOGRAPHIE}

Peyo, « Les schtroumpfs noirs », $1^{\text {ère }}$ série, Ed Dupuis, 1963 ;

Peyo, “The purple smurfs”, Papercut Z Editions, 2010;

Alain, I. « C'est quoi une bande dessinée ? », 2017, Disponible sur : [http://www.lereveil.info/ article-37007967.html], consulté le 13/01/2020 ;

Baron-Carvais. A. « La bande dessinée », Paris, Presses universitaires de France, 1985 ;

Catherine, D. « Les dialogues de BD : Une traduction de l'oral ? » In Bollard, M. « Oralité et traduction », Arras, Artois Presses Université (Collection « Traductologie »), 2001 ;

Chadli, D. « Le texte et le paratexte dans les jardins de lumière et les échelles du levant d'Amin Maalouf », Université de Médéa, Synergie nº 14. 2011 ;

Comitre Narvez, I. « Traduction et créativité dans la bande dessinée : En route pour le Goncourt de J-F. Kierzkowski et M. Ephrem », Université de Malaga. In Cédille revue des études françaises, ISSN-1699-4949, 2015 ;

Epson France Blog Team. « Différences perception des couleurs selon les cultures », 2016, Disponible sur :[https://www.epson.fr/insights/article/differences-de-perception-des-couleursselon-les-cultures], consulté le 25/01/2020 ;

Fatmi, S.E. «Analyser la bande dessinée de la sémiologie à la stripologie », Université de Mascara, Synergie Algérie, $\mathrm{n}^{\circ} 11,2011$;

« Fiche résumé sur les petits hommes bleus ». In super prof ressources. Disponible sur : [https:// www.superprof.fr/ressources/langues/francais/autres-niveaux-fr1/tout-niveau-fr1/ personnages-fiction-enfance.html], consulté le 25/01/2020 ;

Fremion, Y. « Le guide de la bédé francophone », Syros/Aternatives, Paris, 1990 ;

Genette, G. « Seuils », Ed Seuil, Paris, 1987 ;

Gronesteen, T. « La bande dessinée une littérature graphique », Ed les essentiels, Milan, 2005 ;

Harry, M. « Traduire les classiques ? », 2015, Disponible sur :[http://neuviemeart.citebd.org/ spip.php?article961], Consulté le 25/12/2019.

Jose Yuste, F. « Au seuil de la traduction : La paratraduction », In T. Naaijkens (Ed.), Event or incident. On the role of translation in the Dynamics of Cultural Exchange, Oxford, New York, 2010;

Jose Yuste, F. « Traduire l'image dans les albums d'Astérix : A la recherche du pouce perdu en Hispanie ». In B.Bicher, « Le tour du monde d'Astérix ", Actes du colloque tenu à la Sorbonne les 30-31 octobre 2009, Paris, Presses Sorbonne Nouvelle, 2011 ; 
Jose Yuste, F. «Traduire l'image c'est faire de la paratraduction », 2013, Disponible sur : [https:// seuils.hypotheses.org/201], consulté le 12/12/2019 ;

Krajewsky, P. « La quadrature de la bande dessinée », Appareil, 2016, Disponible sur : [http// journals.openedition.org/appareil/2328], consulté le 25/12/2019 ;

Langage-fleur. « Langage des coquelicots », Disponible sur : [http://www.langage-fleurs.com/ coquelicots.htm], consulté le 31/01/2020 ;

Marc, P. «Contraintes et relations de couple : texte/image dans les albums de littérature de jeunesse », Université de Montpellier, Disponible sur : [http://www.ieeff.org/f15parayre.pdf], consulté le 04/01/2020 ;

Razaelle. « Symbolique des couleurs au moyen page », 2006, disponible sur : http:// toros.canalblog.com/archives/2006/09/01/2587361.html, consulté le 19/10/2020 ;

Series. « La signification cachée des couleurs », 2016, disponible sur : [https:// evolutiongraphique.com/la-signification-cachee-des-couleurs-en-communication-visuelle/], consulté le 31/01/2020 ;

Terminologie médicale, dictionnaire en ligne : http://www.bio-top.net/Terminologie/P/ purpura.htm. dernière modification 27/02/2020.

Will, E. « Le récit graphique : narration et bande dessinée », Paris, Vertige graphique, 1998.

\section{NOTES}

1. ${ }^{1}$ Ce terme est employé par Caraballo Laura Cecilia dans sa thèse intitulée « La transposition de la littérature à la bande dessinée : La mise en images chez Alberto Breccia ", soutenue en 2016 à l'Université de Paris Ouest, pour faire référence au récepteur de la bande dessinée qui est à la fois un lecteur et un visionneur (visionner est le fait de regarder avec attention, une série d'images, de documents ou le déroulement d'un film), du support hybridant le graphique au linguistique.

2. ${ }^{2}$ La bande dessinée (vocabulaire). Qu'est ce qu'une bande dessinée (BD), En ligne : https:// ekladata.com/EYfssXEdWTVnOKb3Bw8lCdwC5UQ.pdf

3. ${ }^{3}$ « Gigogne » est employé ici pour vouloir qualifier cet ensemble d'objets qui s'emboitent ou plutôt qui se rassemblent dans un certain ordre pour former un tout complet bien agencé.

4. ${ }^{4}$ Encadré rectangulaire contenant des éléments narratifs et descriptifs assumés par le narrateur, appelés également commentaires.

5. ${ }^{5}$ De courts textes comme "pendant ce temps » ou «le lendemain matin », mais ils peuvent être beaucoup plus détaillés pour expliquer une action ou autre.

6. ${ }^{6}$ La cité internationale de la bande dessinée et de l'image : dossier pédagogique, le texte dans la bande dessinée. En ligne : http://www.citebd.org/IMG/pdf/dossier_peda_texte.pdf

7. ${ }^{7}$ Ce terme est employé par Caraballo Laura Cecilia dans sa thèse sus mentionnée en se référant à la bande dessinée vu la forte présence de combinaisons de textes et d'images.

8. ${ }^{8}$ Professeur titulaire du Département de Linguistique et Traduction à la Faculté de Philologie et Traduction de l'Université Espagnole Vigo, créateur du Groupe de Recherche Traduction \& Paratraduction (T\&P). Traductologue et traducteur professionnel français-espagnol-français, il est l'auteur d'un bon nombre de traductions publiées.

9. ${ }^{9}$ Qui parle directement et de façon explicite du contenu du texte selon Gérard Genette.

10. ${ }^{10}$ " Morphée, inquiète de voir Déméter chercher sans relâche sa fille Perséphone lui offrit un bouquet de coquelicots pour la faire dormir et lui permettre de trouver le soulagement dans l'oubli ». 
11. ${ }^{11}$ Nous retenons dans notre analyse le symbolique de la couleur rouge au moyen âge, parce que toutes les histoires des schtroumpfs se situent dans l'univers du moyen âge, compte tenu des objets, des tenues et des valeurs qu'elles incarnent.

12. ${ }^{12}$ Purpura : du latin purpura, pourpre, relatif à des éruptions de taches rougeâtres sur la peau.

\section{RÉSUMÉS}

La bande dessinée est le genre littéraire le plus célèbre pour son abondance en couples texteimage. En tant que neuvième art, ce genre de «narration figurative (ou le récit en images) » (Yve

Frémion, 1990) aussi agréable qu'il est, possède un mode de lecture assez particulier. L'image dans toutes ses dimensions iconique, plastique et socioculturelle entre en relation intersémiotique avec le texte qui l'accompagne et qui n'est nullement accessoire. La relation entretenue dans ce couple (texte-image) dégage l'essence même du sens à comprendre ensuite à traduire. Dans cette optique, comment le traducteur devrait-il du point de vue de la traductologie, réagir face à cette forte présence d'entités iconotextuelles ? A travers cet article nous essaierons de répondre à cette question parmi d'autres, en se focalisant sur la notion de paratraduction, ce « concept-clé pour traduire le couple texte-image » (Frías, 2010), née à la croisée de la notion de paratexte et la sémiologie de l'image, et qui place méritoirement l'image au sein de l'activité traduisante.

The comic book is the most famous literary genre for its abundant use of text-image couples when narrating. Yve Frémion defines it as "the figurative narration" or (a story in images) (1990). This $9^{\text {th }}$ art has a particular mode of reading. The image with its iconic, plastic and sociolinguistic dimensions undertake an intersemiotic relationship with the text to which it is combined and which is as important as the text. The outcome of this tandem contains the essence of the meaning to be understood and then to translate. From this perspective, how should the translator react, from a traductological point of view, in front of such an abundance of iconotextual entities? Our paper aims at answering this question among others, by focusing on the notion of paratranslation, "this key concept for translating the text-image couple" (Frías, 2010), stemming from the notion of the paratext and the semiology of the image, and which has been given a well-deserved place in translation.

INDEX

Mots-clés : bédé, couple texte-image, paratraduction, traduction, sémiologie de l'image

Keywords : the comic, text-image couple, paratranslation, translation, image semiology

\section{AUTEUR}

\section{BADRA RAMDANI}

Institut de traduction Abu El Kacem Saad Allah, Alger 2 